\title{
Ferramenta Computacional para Estimativa da Dose Efetiva em Exames de Tomografia Computadorizada a partir da Extração de Dados do Cabeçalho DICOM
} Computational Tool for Estimation of Effective Dose in Computed Tomography Exams based on DICOM Header Data Extraction

\author{
Hilton Vicente César ${ }^{1}$, Saulo da Silva Cordeiro², Ester Evelyn Domingues dos Santos², \\ Paulo Mazzoncini Azevedo-Marques ${ }^{3}$
}

${ }^{1}$ Doutorando do Programa de Bioengenharia, Universidade de São Paulo - USP, São Carlos (SP), Brasil

${ }^{2}$ Divisão de Ciências das Imagens e Física Médica do Departamento de Clínica Médica da Faculdade de Medicina de Ribeirão Preto da Universidade de São Paulo - FMRP/USP, Ribeirão Preto (SP), Brasil

${ }^{3}$ Professor Associado da Divisão de Ciências das Imagens e Física Médica do Departamento de Clínica Médica da Faculdade de Medicina de Ribeirão Preto da Universidade de São Paulo - FMRP/USP,

Ribeirão Preto (SP), Brasil

\section{Resumo}

A tomografia computadorizada (TC) é uma técnica que se baseia em raios $\mathrm{X}$ e o seu surgimento no início da década de 1970 provocou uma revolução na medicina. Como consequência do aumento da acurácia, antes não fornecida pela radiografia plana, ocorreu um grande aumento na solicitação desse tipo de exame. Pela literatura, sabe-se que a radiação absorvida no processo de ionização, mesmo em nível diagnóstico, pode ter efeitos carcinogênicos a longo prazo e a TC é um dos exames radiológicos que mais expõem os pacientes a doses elevadas de radiação ionizante. Considerando esse cenário, este artigo apresenta uma ferramenta computacional desenvolvida para possibilitar a avaliação da exposição à radiação ionizante sofrida pelos pacientes a partir da extração de dados do cabeçalho DICOM de exames de TC. Embora existam algumas divergências na literatura em relação ao cálculo dos valores de dose efetiva (DE) a partir de valores obtidos do produto dose-comprimento (DLP), os dados obtidos de uma amostra de 50 pacientes, utilizada para teste do aplicativo desenvolvido, indicam seu potencial para ser uma ferramenta de acompanhamento do nível de exposição de pacientes no ambiente radiológico.

Palavras-chave: tomografia computadorizada; diagnóstico; proteção radiológica; radiação ionizante.

\begin{abstract}
Computed tomography (CT) is an X-rays based technique and its emergence in the early 1970s brought about a revolution in medicine. Because of the increase in accuracy, not previously provided by flat radiography, a large increase occurred in the request for this type of examination. From the literature, it is known that the radiation absorbed in the ionization process, even at the diagnostic level, may have carcinogenic effects in the long term and CT is one of the radiological examinations that most expose patients to high doses of ionizing radiation. Considering this scenario, this article presents a computational tool developed to estimate the exposure level of patients to ionizing radiation based on data extraction from the DICOM header of CT scans. Although there are some divergences in the literature regarding the calculation of effective dose (ED) values from dose-length product (DLP), data obtained from a sample of 50 patients, used to test the developed application, indicate its potential as tool to monitor the level of exposure of patients in the radiological environment.
\end{abstract}

Keywords: tomography; diagnosis; radiation protection; radiation, ionizing.

\section{Introdução}

A tomografia computadorizada (TC) é uma técnica que se baseia em raios $X$ e o seu surgimento no início da década de 1970 provocou uma revolução na medicina ${ }^{1}$. Isso se deve ao modelo de obtenção de imagens tridimensional que possibilita obter imagens claras de órgãos como encéfalo, fígado, rins e pulmões, com a minimização da sobreposição de estruturas anatômicas, impactando positivamente na precisão dos diagnósticos e no acompanhamento de grande número de pacientes. Porém, seu uso intenso e, de certa forma exagerado, faz com que a TC seja um dos maiores responsáveis pela exposição populacional à radiação ionizante devido a procedimentos médicos diagnósticos ${ }^{1}$. Exposição à radiação em procedimentos diagnósticos possui potenciais efeitos deletérios à saúde. Doses em órgãos específicos associadas a exames de TC são comparáveis àquelas de sobreviventes da bomba atômica que receberam baixa dose de radiação e, mesmo assim, tiveram aumento significativo na incidência e mortalidade por câncer ${ }^{2}$.

Embora não existam estudos que comprovem algum tipo de limiar de dose que efetivamente implique em um risco aumentado de desenvolvimento de câncer devido a exames de TC, nem tão pouco exista um consenso na comunidade científica a esse respeito, a atitude prudente do ponto de vista da proteção 
radiológica é considerar que radiação de baixa dose pode causar câncer ${ }^{1}$. Nesse sentido, para se garantir que os benefícios do uso da TC sejam maiores que seus riscos é necessário que se tenha uma forma efetiva de avaliação e acompanhamento da dose recebida pelos pacientes durante os procedimentos diagnósticos de rotina. A Dose Efetiva (DE) foi desenvolvida como um descritor somático de dose que reflete diferenças na sensibilidade dos tecidos biológicos aos efeitos da radiação ionizante. A DE é expressa como a soma das doses equivalentes ponderadas para os diversos tecidos ou órgãos irradiados, representando o risco de todos os efeitos estocásticos para um indivíduo irradiado. Os pesos de ponderação são estabelecidos por um comitê internacional (International Commission on Radiological Protection - ICRP). Uma forma possível de se estimar a DE para um exame de CT é utilizar o produto dose-comprimento (do Inglês, dose-length product - DLP) em conjunto com coeficientes específicos para ajustes de diferenças de idade e regiões do corpo ${ }^{3}$.

Nesse contexto, este artigo apresenta uma ferramenta computacional desenvolvida para possibilitar a estimativa de dose de radiação recebida pelos pacientes em exames de TC, de forma automatizada, a partir da extração de dados do cabeçalho DICOM (do Inglês, Digital Imaging and Communications in Medicine) de exames armazenados em sistemas de arquivamento e distribuição de imagens (do Inglês, Picture Archiving and Communication Systems - PACS) e cálculo da DE a partir de valores de DLP e coeficientes de ponderação específicos, referentes a faixa etária e região anatômica.

\section{Materiais e Métodos}

A ferramenta desenvolvida chamada DICOM Effective Dose (DICOM-ED) é um aplicativo "web" implementado para funcionar como ferramenta auxiliar dentro do fluxo de troca de dados entre os sistemas de informação em saúde no ambiente hospitalar. Ele se utiliza das funcionalidades do padrão DICOM e do protocolo de comunicação HL7 (do Inglês, Health Level 7) para identificar, a partir de uma "worklist", os pacientes para os quais foram solicitados exames de TC, rastrear o banco de dados do PACS, localizar os exames dos pacientes, extrair do cabeçalho dos exames os dados necessários para o cálculo da dose efetiva, armazenar esses dados em um banco não relacional, calcular a dose efetiva de cada exame e a dose acumulada para cada paciente e gerar um conjunto de relatórios pré-definidos. O DICOM-ED foi codificado em linguagem Java, usando o Dcm4che Toolkit, Spring Framework e Grails. O Dcm4che Toolkit é um conjunto de ferramentas de código aberto, implementado em Java, utilizado na área de imagens médicas. A versão do Toolkit utilizado no DICOM-ED foi a dcm4che2 2.0.29. (Dcm4che2 DICOM Toolkit, 2015). Dentre todas as classes existentes neste conjunto de ferramentas, destacam-se para este aplicativo, as classes DcmMWL e DcmQR. A classe DcmMwl é a responsável por fazer a consulta na "worklist" dos exames de TC e identificar os pacientes com exames agendados para um determinado dia. A classe DcmQR é a responsável pela conexão remota com o servidor de arquivamento do PACS. Ela implementa os serviços DICOM C-FIND, C-GET, C-MOVE que possibilitam procurar, recuperar e enviar os exames dos pacientes para o computador no qual o DICOMED está instalado.

O Grails é um framework que foi utilizado para codificação rápida em alto nível e o Spring foi utilizado para fazer a comunicação com o banco de dados usado no aplicativo. Por questão de flexibilidade de configuração, considerando possíveis ajustes futuros no aplicativo, foi escolhido o banco de dados não relacional MongoDB versão 3.2 para armazenamento dos dados extraídos dos cabeçalhos DICOM dos exames e os resultados dos cálculos da DE.

Na sintaxe do padrão DICOM a "tag" referente ao DLP é uma tag privada. Cada fabricante possui uma tag específica para esse fim. O equipamento de TC utilizado neste estudo foi da marca Philips Medical Systems, modelo Brilliance Big Bore, disponível no Serviço de Radiodiagnóstico do Centro de Ciências das Imagens e Física Médica do Hospital das Clínicas da Faculdade de Medicina de Ribeirão Preto, da Universidade de São Paulo (CCIFM/HCFMRP). Nesse equipamento a tag que armazena o valor do DLP é a $(00 E 1,1021)$. Um valor do DLP é gerado para cada série de imagens e essa "tag" pode se repetir, pois o exame pode ter uma ou mais séries. A última ocorrência desta "tag" no cabeçalho DICOM representa o somatório dos valores de DLP do exame (DLP Total). Neste trabalho, foi utilizado o valor extraído dessa última ocorrência. A lista de "tags" cujos valores foram extraídos para cada exame e armazenados no banco de dados está apresentada na Tabela 1.

Tabela 1. "Tags" cujos valores foram extraídos para cada exame e armazenados no banco de dados

\begin{tabular}{ll}
\hline Tag do DICOM & \multicolumn{1}{c}{ Informação } \\
\hline$(0010,0010)$ & NamePatient \\
$(0010,0020)$ & IdPatient \\
$(0010,0040)$ & SexPatient \\
$(0010,0030)$ & BirthDatePatient \\
$(0010,1010)$ & PatientAge \\
$(0018,1030)$ & ProtocolName \\
$(0008,1030)$ & StudyDescription \\
$(0008,0020)$ & StudyDate \\
$(0008,0030)$ & StudyTime \\
$(0018,0060)$ & Kvp \\
$(0020,000 D)$ & IdStudy \\
$(00 \mathrm{E} 1,1021)$ & DLP \\
\hline
\end{tabular}

A partir dos valores extraídos das "tags" foi feito o cálculo da DE (mSv) como sendo o produto do DLP Total (mGy.cm) por um fator de ponderação de dose efetiva normalizada $\mathrm{K}$ (em mSv.mGy $\left.{ }^{-1} \cdot \mathrm{cm}^{-1}\right)$. Os coeficientes de ponderação foram definidos por faixa etária e região anatômica ${ }^{4}$ e estão apresentados na Tabela 2. 
Para um teste inicial do DICOM-ED foram feitas as estimativas de dose para os pacientes com exames relacionados na "worklist" para a rotina de TC do dia 01/02/2018 do CCIFM/HCFMRP, totalizando 50 pacientes. Os valores de DE obtidos para os exames de cada paciente existentes no PACS, assim como os valores acumulados para cada um deles, de uma amostragem de 25 pacientes foram calculados e confirmados manualmente.

Tabela 2. Coeficientes de ponderação definidos por faixa etária e região anatômica ${ }^{4}$

\begin{tabular}{|c|c|c|}
\hline Região Anatômica & Faixa Etária & Coeficiente \\
\hline \multirow{4}{*}{ ABDOME } & 1 a 5 & 0.0300 \\
\hline & 5 a 10 & 0.0200 \\
\hline & 10 a 20 & 0.0150 \\
\hline & $>20$ & 0.0150 \\
\hline \multirow{4}{*}{ TORAX } & 1 a 5 & 0.0260 \\
\hline & 5 a 10 & 0.0180 \\
\hline & 10 a 20 & 0.0130 \\
\hline & $>20$ & 0.0140 \\
\hline \multirow{4}{*}{ CRANIO } & 1 a 5 & 0.0067 \\
\hline & 5 a 10 & 0.0040 \\
\hline & 10 a 20 & 0.0032 \\
\hline & $>20$ & 0.0021 \\
\hline \multirow{4}{*}{ MAMA } & 1 a 5 & 0.0087 \\
\hline & 5 a 10 & 0.0057 \\
\hline & 10 a 20 & 0.0042 \\
\hline & $>20$ & 0.0031 \\
\hline \multirow{4}{*}{ CERVICAL } & 1 a 5 & 0.0120 \\
\hline & 5 a 10 & 0.0110 \\
\hline & 10 a 20 & 0.0079 \\
\hline & $>20$ & 0.0059 \\
\hline \multirow{4}{*}{ PELVE } & 1 a 5 & 0.0300 \\
\hline & 5 a 10 & 0.0200 \\
\hline & 10 a 20 & 0.0150 \\
\hline & $>20$ & 0.0150 \\
\hline \multirow{4}{*}{ ORBITA } & 1 a 5 & 0.0085 \\
\hline & 5 a 10 & 0.0057 \\
\hline & 10 a 20 & 0.0042 \\
\hline & $>20$ & 0.0031 \\
\hline
\end{tabular}

\section{Resultados}

O fluxo de dados do DICOM-ED está apresentado na Figura 1. A ordem de execução das etapas é dada pelas numerações atribuídas as classes.

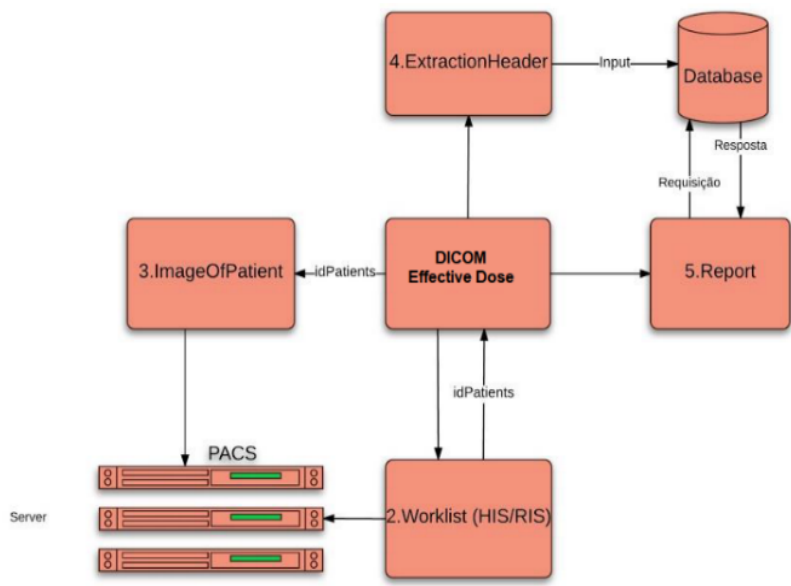

Figura 1. Representação esquemática do fluxo de dados no aplicativo DICOM-ED ${ }^{5}$.
A partir dos dados apresentados na Tabela 1 e com os valores de DE calculados o aplicativo pode gerar um conjunto de relatórios. Por exemplo, exibir as informações referentes aos exames de um determinado paciente, incluindo datas de realização e tipos do exame, DLP de cada exame, DLP acumulada em todos os exames encontrados, DE de cada exame e DE efetiva acumulada (Figura 2). $\mathrm{O}$ aplicativo também pode mostrar dados na forma de gráficos, por exemplo, um gráfico mostrando a quantidade de exames referentes a cada protocolo de aquisição em um determinado período de tempo (Figura 3), entre outros. A Tabela 3 apresenta as doses efetivas, o número de exames, o protocolo e data de cada um, para quatro pacientes escolhidos aleatoriamente no banco de dados.

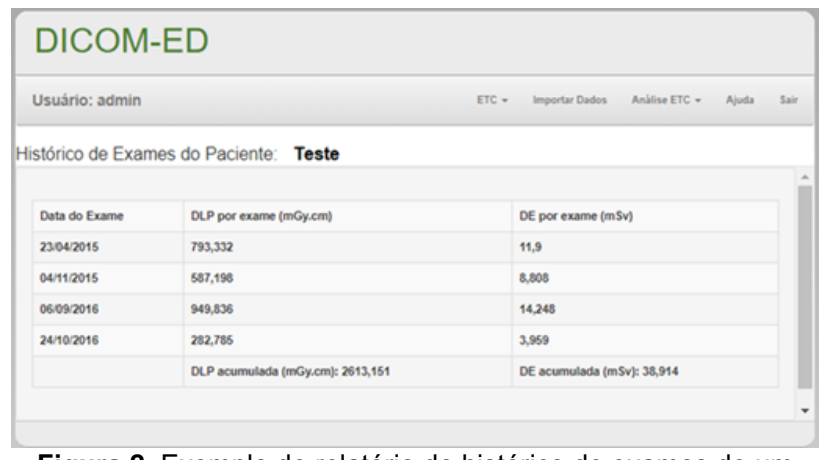

Figura 2. Exemplo de relatório de histórico de exames de um paciente, indicando o conjunto de exames, com suas respectivas datas e DE e a DE acumulada pelo paciente até o último exame (o nome do paciente foi substituído pela palavra Teste para fins de anonimização)

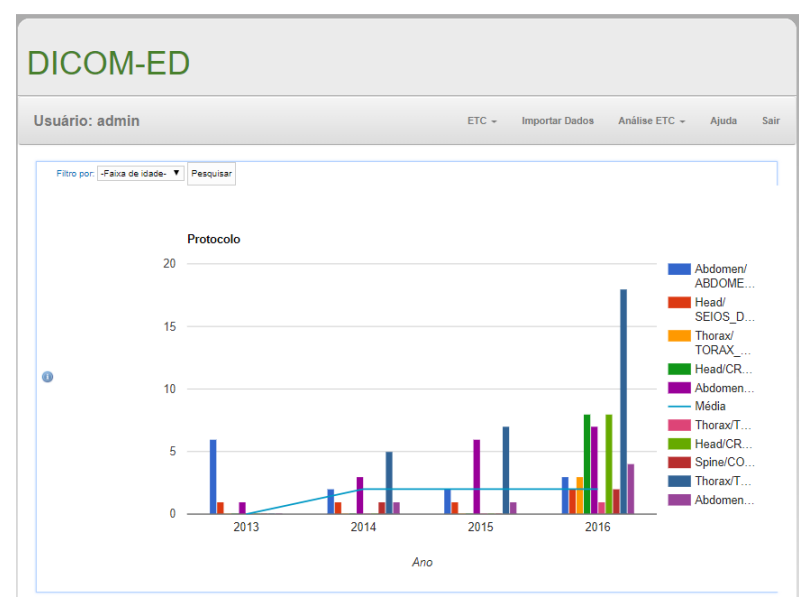

Figura 3. Exemplo de gráfico mostrando a quantidade de exames referentes a cada protocolo de aquisição em um determinado período de tempo, considerando os pacientes existentes na worklist do dia 01/02/2018.

Tabela 3. Exemplo de relatório mostrando os exames e doses efetivas para quatro pacientes do conjunto de teste.

\begin{tabular}{|c|c|c|c|}
\hline Paciente & $\begin{array}{l}\text { DE por } \\
\text { exame } \\
(\mathrm{mSv})\end{array}$ & $\begin{array}{l}\mathrm{N}^{\circ} \\
\text { Exames }\end{array}$ & Protocolo/Data \\
\hline \multirow{4}{*}{1} & 5,885 & \multirow{4}{*}{4} & $\begin{array}{l}\text { Abdomen/ABDOMEN_ROTINA } \\
\text { _HC S/CONT - 24/10/2016 }\end{array}$ \\
\hline & 6,549 & & $\begin{array}{l}\text { Abdomen/ABDOMEN_ROTINA } \\
\text { _C/ FASE_45 - 11/03/2013 }\end{array}$ \\
\hline & 3,803 & & $\begin{array}{l}\text { Abdomen/ABDOMEN_ROTINA } \\
\text { _HC_S/CONT - 26/02/2013 }\end{array}$ \\
\hline & 4,101 & & Abdomen/ABDOMEN_ROTINA \\
\hline
\end{tabular}




\begin{tabular}{|c|c|c|c|}
\hline \multirow{4}{*}{2} & & & _HC_S/CONT - 17/06/2010 \\
\hline & 1,187 & \multirow{3}{*}{3} & $\begin{array}{l}\mathrm{Head} / \text { SEIOS_DA_FACE } \\
\text { ROTINA_HC- } 21 / 11 / 2013\end{array}$ \\
\hline & 3,696 & & $\begin{array}{l}\text { Thorax/TORAX_ALTA } \\
\text { RESOLUCAO HC - } 24 / 10 / 2016\end{array}$ \\
\hline & 2,438 & & $\begin{array}{l}\text { Thorax/ALTA_RESOLUCAO } \\
\text { _ROTINA_HC }-17 / 05 / 2010\end{array}$ \\
\hline \multirow[b]{2}{*}{3} & 2,448 & \multirow[b]{2}{*}{2} & $\begin{array}{l}\text { Thorax/TORAX_ROTINA_HC } \\
\text { C/C - 24/10/2016 }\end{array}$ \\
\hline & 16,285 & & $\begin{array}{l}\text { Abdomen/ABDOMEN_- } \\
\text { TRIFASICO_BOLUS - } \\
\text { 24/10/2016 }\end{array}$ \\
\hline \multirow{2}{*}{4} & 2,281 & \multirow{2}{*}{2} & $\begin{array}{l}\text { Head/CRANIO_ROTINA_Cl } \\
\text { CONTRAST }-24 / 10 / 201 \overline{6}\end{array}$ \\
\hline & 0 & & $\begin{array}{l}\text { Spine/COL._LOMBAR_ } \\
\text { ROTINA - 18/02/2014 }\end{array}$ \\
\hline
\end{tabular}

\section{Discussão}

Dose Efetiva foi definida pela ICRP como uma quantidade única de dose que reflita o risco global de efeito deletério quando uma pessoa é exposta à radiação ionizante. Seu uso principal é como ferramenta para se buscar a conformidade com limites de dose para proteção de trabalhadores ocupacionalmente expostos e do público em geral, mas também pode ser útil para se comparar riscos associados a diferentes técnicas e procedimentos de obtenção de imagens para um determinado paciente. Porém, não deve ser utilizada para a avaliação precisa de riscos de pacientes individuais ${ }^{6}$.

Nesse contexto, embora existam algumas divergências na literatura em relação ao cálculo dos valores de DE a partir do $D^{2} P^{3}$, particularmente devido à grande variedade de técnicas, geometrias de exposição, variações anatômicas e de idade inerentes à realização dos exames, o uso de coeficientes de ponderação de dose efetiva normalizada reconhecidos e divulgados pela comunidade científica pode fornecer estimativas de DE suficientemente precisas para avaliação e padronização de protocolos e referência de exposição de pacientes ${ }^{5}$. Nesse sentido, o trabalho aqui apresentado traz como contribuição principal a implementação de uma ferramenta automatizada que possibilita rastrear os exames existentes em um PACS, pertencentes a pacientes presentes em uma "worklist" de exames de CT e calcular as doses efetivas relativas a cada exame e o acumulado de dose para cada paciente, a partir de dados extraídos do cabeçalho DICOM.

O aplicativo possibilita, então, a geração de diferentes tipos de tabelas e gráficos para fins de gerenciamento de doses de pacientes e de organização do serviço de diagnóstico por imagem. Como limitações principais, destacam-se o fato da atual versão do DICOM-ED funcionar somente para equipamentos que utilizem a "tag" $(00 \mathrm{E} 1,1021)$ para armazenamento dos valores do DLP e a necessidade de se buscar a atualização dos valores dos coeficientes de ponderação de dose efetiva normalizada utilizados no calcula da DE. Essas limitações deverão ser equacionadas em versões futuras do DICOM-ED.

\section{Conclusões}

Foi apresentado um aplicativo "web" (DICOM-ED) que possibilita calcular e disponibilizar de forma automatizada uma estimativa da dose efetiva de exames de CT existentes em um PACS, bem como a dose efetiva total acumulada, referentes a pacientes relacionados em uma "worklist" que esteja em conformidade com o protocolo DICOM. Os resultados iniciais mostram bom potencial de uso do aplicativo para avaliação da exposição de pacientes submetidos a exames de TC de rotina. Como desenvolvimento futuro propõe-se a integração dos relatórios fornecidos pelo DICOM-ED no registro eletrônico dos pacientes do Hospital das Clínicas da Faculdade de Medicina de Ribeirão Preto.

\section{Agradecimentos}

À CAPES (Coordenação de Aperfeiçoamento de Pessoal de Nível Superior) ao CNPq (Conselho Nacional de Desenvolvimento Científico e Tecnológico) e à FAPESP (Fundação de Amparo à Pesquisa do Estado de São Paulo) pelo apoio financeiro.

\section{Referências}

1. Semelka RC, Armao DM, Elias Junior J, Uda W. Imaging Strategies to Reduce the Risk of Radiation in CT Studies, Including Selective Substitution With MRI. J. Magn. Reson. Imaging 2007; 25:900-909.

2. Brenner DJ, Elliston CD. Estimated radiation risks potentially associated with full-body CT screening. Radiology 2004; 232:735-738.

3. Brady SL, Mirro AE, Moore BM, Kaufman RA. How to Appropriately Calculate Effective Dose for CT Using Either Size-Specific Dose Estimates or Dose-Length Product. AJR 2015; 204:953-958.

4. Shrimpton P C 2004 Assessment of patient dose in CT NRPB Report no NRPB-PE/1/2004 (Chilton: National Radiological Protection Board).

5. Santos, EED. Trabalho de Conclusão de Curso (Graduação em Informática Biomédica) - Universidade de São Paulo. Estudo de um algoritmo para extração de dados do cabeçalho DICOM de exames de Tomografia Computadorizada para a avaliação da exposição à radiação ionizante dos pacientes, 2016.

6. Shrimpton PC, Wall BF. Effective Dose and Dose-Length Product in CT. Radiology 2009; 2:604 (Letter to the editor).

\section{Contato:}

Prof. Dr. Paulo Mazzoncini de Azevedo Marques

Centro de Ciências das Imagens e Física Médica

Hospital das Clínicas da Faculdade de Medicina de Ribeirão Preto - USP

Av. dos Bandeirantes 3900, Campus USP - Monte Alegre. 14049-900, Ribeirão Preto - SP.

pmarques@fmrp.usp.br 